\title{
In-line ATR-UV and Raman Spectroscopy for Monitoring API Dissolution Process During Liquid-Filled Soft-Gelatin Capsule Manufacturing
}

\author{
Boyong Wan, ${ }^{1,2}$ Christopher A. Zordan, ${ }^{1}$ Xujin Lu, ${ }^{1}$ and Gary McGeorge ${ }^{1}$
}

Received 20 September 2015; accepted 16 November 2015; published online 24 November 2015

\begin{abstract}
Complete dissolution of the active pharmaceutical ingredient (API) is critical in the manufacturing of liquid-filled soft-gelatin capsules (SGC). Attenuated total reflectance UV spectroscopy (ATR-UV) and Raman spectroscopy have been investigated for in-line monitoring of API dissolution during manufacturing of an SGC product. Calibration models have been developed with both techniques for in-line determination of API potency. Performance of both techniques was evaluated and compared. The ATR-UV methodology was found to be able to monitor the dissolution process and determine the endpoint, but was sensitive to temperature variations. The Raman technique was also capable of effectively monitoring the process and was more robust to the temperature variation and process perturbations by using an excipient peak for internal correction. Different data preprocessing methodologies were explored in an attempt to improve method performance.
\end{abstract}

KEY WORDS: ATR-UV; in-line monitoring; Raman; soft-gelatin capsule.

\section{INTRODUCTION}

The soft-gelatin capsule (SGC) is a common oral dosage form, which consists of a gelatin-based shell surrounding a liquid fill solution. Compared with other solid dosage forms, SGC products offer several benefits, including enhanced bioavailability, improved content uniformity $(\mathrm{CU})$ for low-dose drugs, good product stability, etc. (1-3). Because of these benefits, the development of SGC products is of increasing interest in recent years. SGC manufacturing usually includes several steps. Firstly, solid API is introduced to the excipient solution (usually nonaqueous) in a vessel and dissolved to form a homogeneous liquid fill solution. In the second step the gelatin shell materials to be used for solution encapsulation are prepared. The third step is encapsulation, which brings the gelatin shell and the fill material together to form SGCs. In this step, two flat ribbons are made that form the shell material and they are brought together on a die of the desired shape and size, and a pump delivers a precise amount of fill solution to the shell. A drying step follows the encapsulation, which may last a number of days before proceeding to washing/roller-grading and packaging (2-4).

During this manufacturing the API mixing and dissolution is a critical process parameter since it will have a direct impact on the product potency, content uniformity (CU), in vivo absorption and stability as well as drug exposure in vivo. For example, undissolved API may nucleate the re-

\footnotetext{
${ }^{1}$ Analytical and Bioanalytical Development, Bristol-Myers Squibb Co., 1 Squibb Drive, New Brunswick, New Jersey 08901, USA.

${ }^{2}$ To whom correspondence should be addressed. (e-mail: boyong.wan@bms.com)
}

crystallization of the product over time and thus change the dissolution profile upon stability. Consequently, it must be assured that API was completely dissolved and the solution is at an acceptable potency level (5). Traditionally, this is achieved by frequent manual sampling of the fill solution which is followed by analytical testing using offline HPLC analysis. In addition, the process may mitigate the risk of incomplete dissolution by introducing excessively long dissolution/mixing times. This type of offline testing procedure is labor-intensive and time-consuming, resulting in a less than optimal feedback to the process and in addition the offline analysis provides little information regarding the API dissolution dynamics in the mixing process, particularly when API powder properties, such as particle size, density, morphology, etc., are varying batch to batch.

Quality be design (QBD) (6) has been becoming the new norm in pharmaceutical development as it aims for better process understanding and improved product quality control. Process analytical technology (PAT) (7) has been an integrated part for successful implementation of QBD principles as it makes real-time monitoring, feedback and control a possibility. With the increasing acceptance of QBD/PAT initiatives by the pharmaceutical industry, real-time analytical tools are more frequently used for improving process understanding, implementing process control, and product release. For example, NIR spectroscopy has been widely used for online/at-line analysis of solid dosage drug product in manufacturing (8-11). A number of publications have shown the use of Raman for in-process monitoring and control during both drug substance and product manufacturing $(8,12-14)$. However, to our knowledge, no applications have been reported on using in-line spectroscopy tools to support manufacturing of SGC products. 
Considering the risk factors associated with the SGC manufacturing and current practice for analysis, it would be beneficial to implement in-line monitoring and analysis in the API dissolution step.

UV spectroscopy has been widely used in the pharmaceutical industry for the assessment of API potency, dissolution testing of dosage forms, and cleaning validation in a laboratory environment $(15,16)$. Traditionally this is done by bringing manually prepared samples to UV spectrometer or by interfacing the UV detector with a high-performance liquid chromatography (HPLC) equipment. In recent years, advances in highquality optical fibers and sensitive array detectors have made in situ UV sampling and analysis possible by "bringing the UV spectrometer to the sample". This has greatly simplified sampling and improved analytical efficiency (17). Applications have been reported using fiber-optics based UV probes of different geometry for in situ dissolution testing and for testing the cleaning of manufacturing vessels (17-22). In these studies, transflectance or transmission probes were typically employed that can be inserted into the sample solution directly. The quality of UV measurements using such in situ transflectance/ transmission probes can be degraded by suspended particles and bubbles, although some of such interference may be overcome by operational changes and mathematical data treatment (17). Another limitation of such probes is that they are only suitable for the measurement of lower concentration samples, such as for drug product dissolution testing in aqueous media, because of the available pathlength of the probes. Typically these probes have a minimum pathlength of 1-2 mm (17), which often makes UV absorbance measurement impossible for highconcentration or highly absorbing samples. Attenuated total reflectance (ATR) probes represent another type that can be used for spectroscopic measurements of high-concentration samples, and they are more tolerant of bubbles and/or particles in the sampling environment. In comparison with the transmittance and transflectance probes, the ATR probe does not have a mirror or sample gap making it ideal for direct insertion into a manufacturing process stream. As examples, ATR-UV monitoring has been applied to support crystallization processes $(23,24)$ and ATR-FTIR has been used for the in-line process monitoring of large-scale organic reactions (25-27). The phenomenon of ATR is based on the behavior of incident light crossing the interface between a transparent material with high refractive index, $\mathrm{n} 1$ (the crystal of the ATR probe) and a transparent medium with a lower refractive index, $\mathrm{n} 2$ (the sample) (23). A portion of the light escapes the crystal (the evanescent wave) and propagates into the sample layer at the crystal surface. The effective pathlength of the photons is only a few microns for UV wavelengths and as a result of this short pathlength, ATR-UV can be applied to highly absorbing solutions, as well as highly concentrated sample solutions, on which standard transmission or transflectance measurements are not feasible.

In ATR measurements, the effective path length and consequently absorbance intensity is related to several factors including incident angle, wavelength of incident light, and the refractive index of both ATR material and sample. While the refractive index of the ATR crystal does not vary significantly the refractive indices of solutions change with sample conditions. The refractive index of a solution changes with solution concentration and temperature (28) and the impact of such change needs to be carefully evaluated for specific PAT application.
Raman spectroscopy has been found in a wide array of applications in the pharmaceutical industry, including inprocess monitoring of various processes such as synthetic organic reactions, API crystallization, granulation and coating $(12,24,29,30)$. The high specificity of Raman spectral features makes it a suitable tool for analyzing multi-component systems both qualitatively and quantitatively. In addition, Raman has low interference from water that makes it ideal for analysis of aqueous solutions. It has been used for in situ monitoring of drug dissolution kinetics during drug product development (31-33). However, since Raman spectroscopy is essentially a single-beam technique, Raman signal intensity can be liable to instrumental variations and changes in sampling conditions as well. Contributions due to the instrument or sampling variations must be considered when doing absolute quantitative analyses. For these reasons, it is common to measure concentrations of the components in a mixture relative to an internal reference band.

In this work, both ATR-UV and Raman spectroscopy were investigated for in-line process monitoring and quantitative determination of API potency during mixing of API in manufacturing of a BMS SGC product (20\% drug loading). Performance of the two techniques was evaluated and compared. Methodology for improvement of the robustness of the in situ measurement is addressed in the discussion.

\section{MATERIALS AND METHODS}

\section{Raman}

A Kaiser RXN2 hybrid Raman spectrometer (Ann Arbor, MI) equipped with a 785-nm laser was used. The Raman spectrometer was coupled with an 18-in Hastelloy immersion probe ( 0.5 in OD) (Kaiser Optical Systems, Inc., Ann Arbor, MI) for data collection in solution. A 2-s exposure time was used and 10 acquisitions were averaged for each spectrum to increase signal to noise. One such composite Raman spectrum was collected each minute during the API dissolution process. Raman data collection employed the IC Raman (version 4.1, Kaiser Optical Systems, Ann Arbor, MI) software package.

\section{ATR-UV}

A Leap Technologies OPT-DISS UV spectrometer (LEAP Technologies, Carrboro, NC) equipped with a single channel stainless steel ATR fiber-optic probe (661.812-UV, Hellma GmbH \& Co., Germany) was used. The probe has a dimension of $12.7 \mathrm{~mm}$ in diameter and $200 \mathrm{~mm}$ in length. It uses a three-bounce sapphire ATR element with a Kalrez ${ }^{\circledR}$ seal that is resistant up to 15 bar and $150^{\circ} \mathrm{C}$. Background ATR-UV spectra were collected for the excipient solution prior to the addition of the API to the vessel. After initiation of API dissolution, UV absorbance spectra were collected once every $30 \mathrm{~s}$. An exposure time of $10 \mathrm{~ms}$ was used for each spectrum collection.

Data analysis was performed using TQ Analyst (Version 3 SP6, Thermo Fisher Scientific Inc., Madison, WI). Both ATR-UV and Raman data were exported to TQ Analyst, in which subsequent data processing and model development were implemented. 


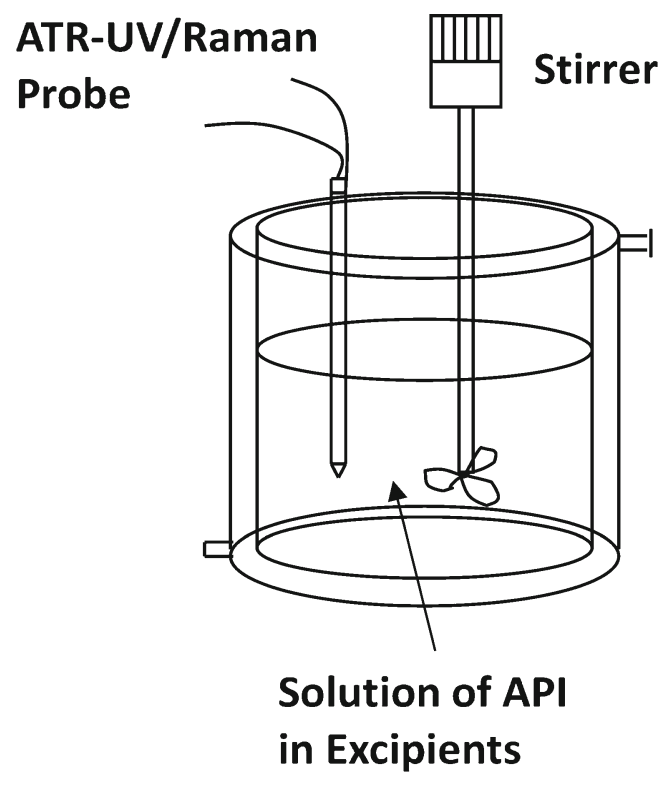

Fig. 1. Experimental setup for UV and Raman data collection

\section{Refractometer}

A Digital ATAGO PAL-RI refractometer (ATAGO Co. Ltd, Tokyo, Japan) was used for measuring the refractive index of the solution samples.

\section{SGC Formulation and Manufacturing}

The SGC product investigated in this study contains $20 \%$ (wt $\%$ ) of API in the fill solution consisting of liquid excipients that include solubilizers and surfactants. Two different experimental setups were employed in this work. Firstly, feasibility studies and model calibration were performed at small scale, in vessels that simulated the commercial equipment, where the Raman probe and ATR-UV probe were held by clamps and inserted in the mixing vessel with an open top. Secondly, batch monitoring within pilot scale and commercial equipment, where the Raman probe and ATR probes were integrated into the sealed mixing vessel through the top via special connectors. During mixing, the API powder was added to a container filled with pre-weighted amount of liquid excipient solution of fixed composition. The small scale experimental setup is as shown in Fig. 1. A container filled with excipient solution was used as the dissolution tank. The ATR-UV and Raman probes were inserted into the solution. During batch manufacturing, a predetermined amount of excipient solution was first added to the container, and then weighed amount of API was gradually added to the excipients solution under stirring conditions.

\section{RESULTS AND DISCUSSION}

\section{Calibration Data Collection}

In order to develop quantitative methods, calibration samples at five different potency levels were prepared by running small scale experiments of around $200 \mathrm{~g}$ batch size. The five levels were 80, 90, 100, 110 and $120 \%$ of label claim potency. All calibration experiments were implemented under ambient lab conditions. In each experiment, a pre-weighed amount of API was gradually added to the dissolution tank filled with fixed amount of excipient solutions. ATR-UV and Raman data were collected continuously starting upon introduction of the API. However, only data collected after the API dissolution was completed were used for calibration purposes.

\section{Univariate Calibration Development Using ATR-UV}

Figure 2 shows representative ATR-UV calibration spectra of the five different levels, which clearly demonstrates a monotonic absorbance increase corresponding to the changes in potency or concentration. Traditionally, UV quantitative

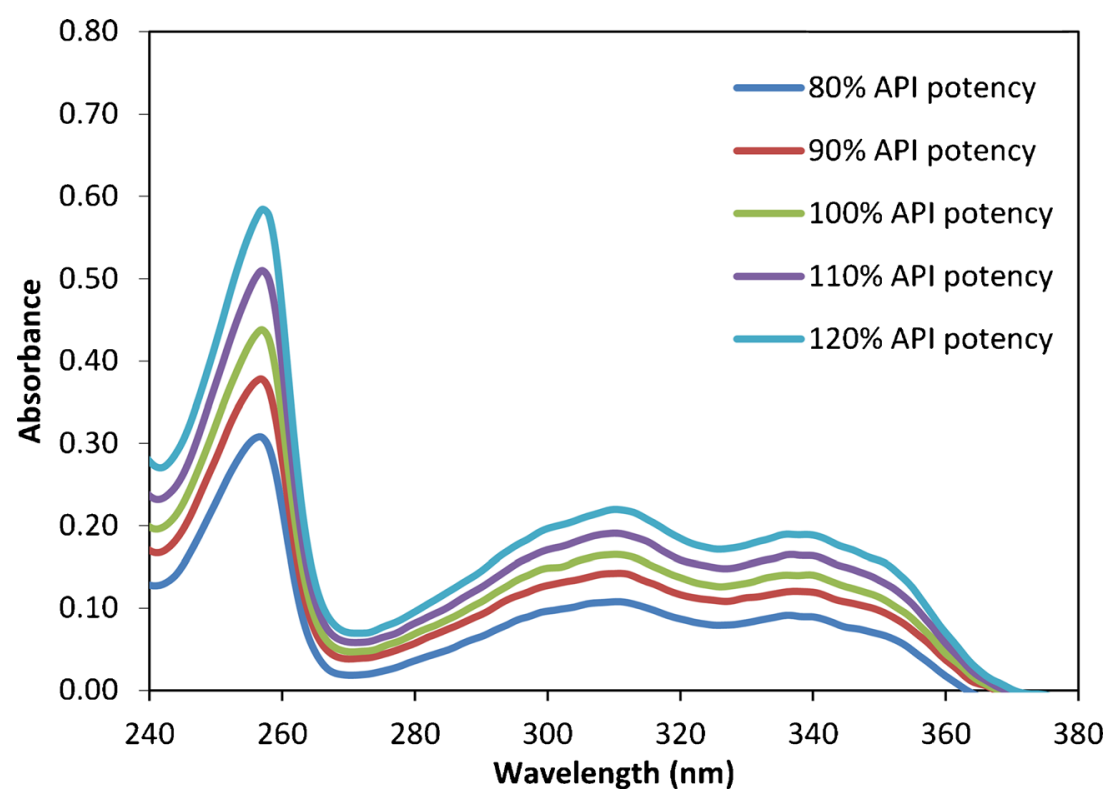

Fig. 2. ATR-UV absorbance spectra of five levels of calibration samples 


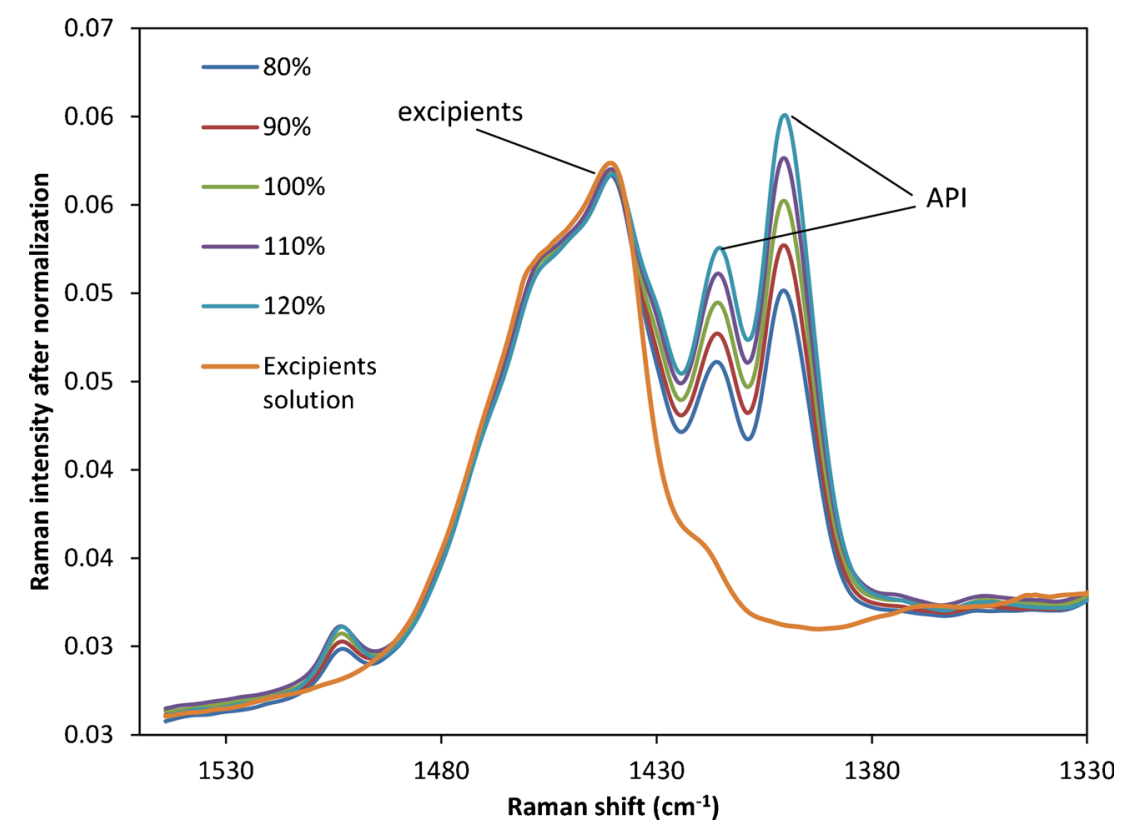

Fig. 3. Raman spectra of calibration samples and excipient solution. All spectra were processed with normalization by using peak area of excipients

analysis is often implemented with simple univariate calibration. When a single wavelength was used for quantitative calibration, the UV absorbance intensity value at $340 \mathrm{~nm}$ was calculated for all calibration spectra after a baseline correction through subtraction. A linear relationship was then established between API concentration and baseline corrected absorbance intensity at $340 \mathrm{~nm}$, with $R^{2}$ above 0.99. This indicated that the univariate analysis provides a high degree of correlation and may prove a suitable tool for monitoring the API dissolution.

\section{Raman Calibration and Method Development}

Figure 3 shows representative Raman spectra of the five calibration sample solutions and excipient solution. The API has two peaks around 1400 and $1415 \mathrm{~cm}^{-1}$, which can be used for calibration development. The excipients have broad but intense peak around $1440 \mathrm{~cm}^{-1}$.

During early development of the Raman method, the intensity of the API peak at $1415 \mathrm{~cm}^{-1}$ was utilized for monitoring the API dissolution and mixing process. The API peak intensity at $1415 \mathrm{~cm}^{-1}$ was calculated by using a two-point (1380 and $1492 \mathrm{~cm}^{-1}$ ) baseline correction. However, a dissolution profile obtained for a small (200 g) experimental batch using this peak with such calculation showed significant fluctuations and drifts (see Fig. 4). The profile was recorded from the beginning when the API was added under stirring condition. A significant drop of the profile after approximately $28 \mathrm{~min}$ was observed. Similar degree of intensity drop was also observed on peaks at other spectral locations. It was unclear what exactly caused this peak intensity drop. Possible reasons may include process interruption or air bubble formation, which may have resulted in a change of the

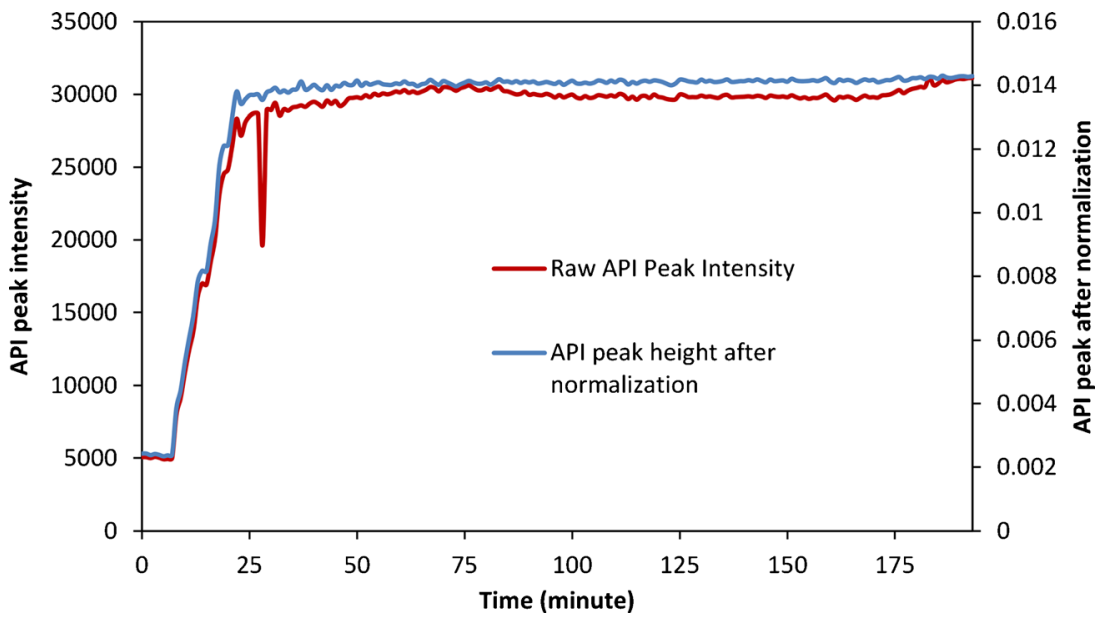

Fig. 4. Peak intensity profile of API during dissolution of a small experimental batch 


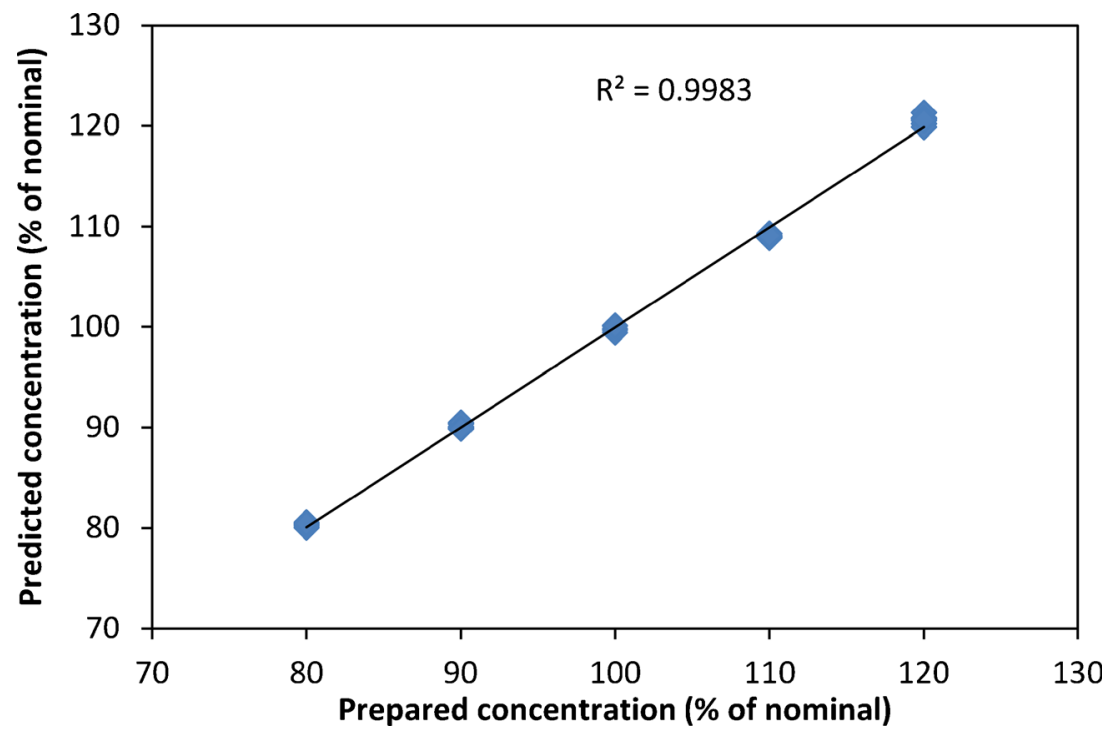

Fig. 5. Calibration correlation plot between prepared API concentration and predicted API concentration by Raman method

sampling conditions around the Raman probe. In addition, instrumental drift due to causes such as variations of laser power will also correspondingly impact raw Raman signal intensity.

An alternative approach was evaluated by leveraging the excipient solution background response as an internal calibration or normalization. Since the excipient solution has a strong peak in the sample spectra and the amount of excipient in the solution doesn't change during mixing, the raw Raman spectra can be normalized by using the excipient features to correct such Raman intensity variations. In this approach, the excipient peak area in each Raman spectrum in the range of 1425 and $1491 \mathrm{~cm}^{-1}$ (after a two-point baseline correction) was calculated and used as a normalization factor. After the normalization preprocessing, the API Raman intensity profile of the same run yielded a smooth profile and the process distortion was negated as shown in Fig. 4. Clearly, the normalization procedure can correct such variations in Raman intensity.
With the model feasibility established a partial least squares (PLS) multivariate calibration model was then developed for the quantitative analysis of the API dissolved in the SGC fill solution. To establish the model, after normalization using excipients peak area by the procedure described above, the Raman spectra were processed with a 9-point SavitzkyGolay first derivative and the spectral range of 1376 to $1430 \mathrm{~cm}^{-1}$ was used. With one PLS factor, a root mean squared method error of calibration (RMSEC) was estimated to be $0.6 \%$ and the root mean squared cross-validation error (RMSECV) using a leave one out approach was $0.84 \%$. The calibration correlation plot is shown in Fig. 5.

\section{Comparison of the ATR-UV and Raman Methods}

After the preliminary method development, both the ATR-UV and Raman models were used for inline monitoring of a 2-kg experimental scale-up batch (with a target concentration of $\sim 90 \%$ of label claim). The run was conducted

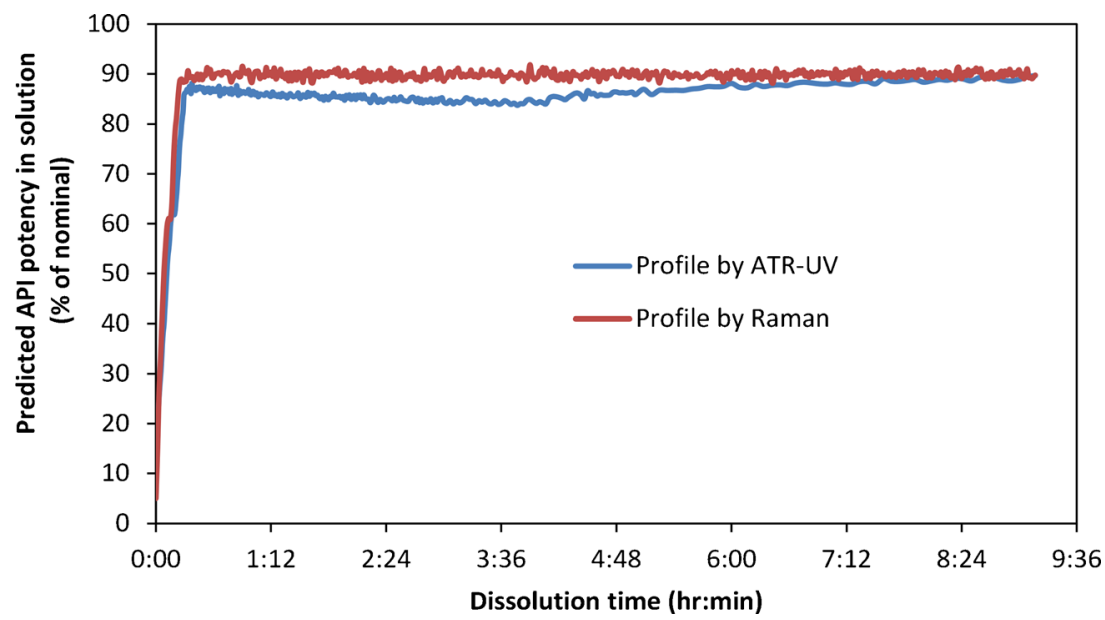

Fig. 6. API dissolution profiles as predicted by ATR-UV and Raman method on an experimental scale-up batch 


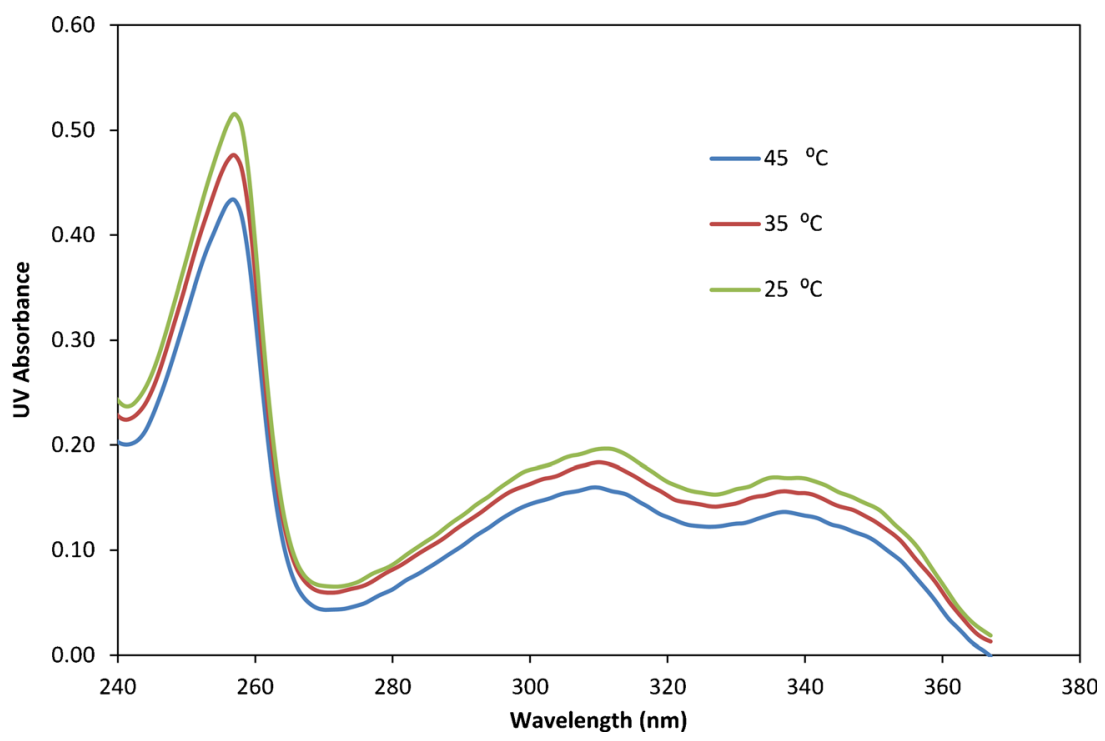

Fig. 7. ATR-UV spectra of API solution $(\sim 100 \%$ label claim) under three different temperatures

overnight under ambient lab conditions for approximately 10 h. Figure 6 displays the predicted potency profile by the two methods. At the beginning, profiles by both methods appear to be very similar, showing quick dissolution of API. During the mixing process, the Raman profile appeared to be very consistent throughout the whole process. However, there was some drift in the ATR-UV profile, where the prediction first gradually went down and then came back to level later on.

The cause of the drift in the dissolution profile measured by the ATR-UV was investigated and it was found that the temperature changes during the run could be the major source of the problem. When a high speed impeller (1600 rpm) was used in a relatively small dissolution vessel (holding a 2-kg solution), the revolution of the impeller could generate heat in the vessel, causing a temperature change during the mixing process. Experiments were designed to evaluated possible impact of temperature on the ATR-UV method. In this evaluation, the API solutions of three different levels, 80, 100, and $120 \%$ of label claim were measured by ATR-UV under different controlled temperature conditions. The spectra of $100 \%$ solution at 25,35 , and $45^{\circ} \mathrm{C}$ are shown in Fig. 7. As can be seen, the absorbance decreases significantly with increasing temperature $(\sim 10 \%$ with temperature increasing from 25 to $45^{\circ} \mathrm{C}$ ). Similarly, Fig. 8 shows changes of UV absorbance intensity $(340 \mathrm{~nm})$ with temperature for all three solutions, which are in a similar trend. However, it also can be found through careful observation that there is some slight difference in the degree of slope for the three solutions, indicating that API concentration may also play a role. The solution of $120 \%$ potency appears to have a steeper slope in absorbance change with corresponding temperature change.

It was assumed that these absorbance changes might be due to density changes resulting from volume change with

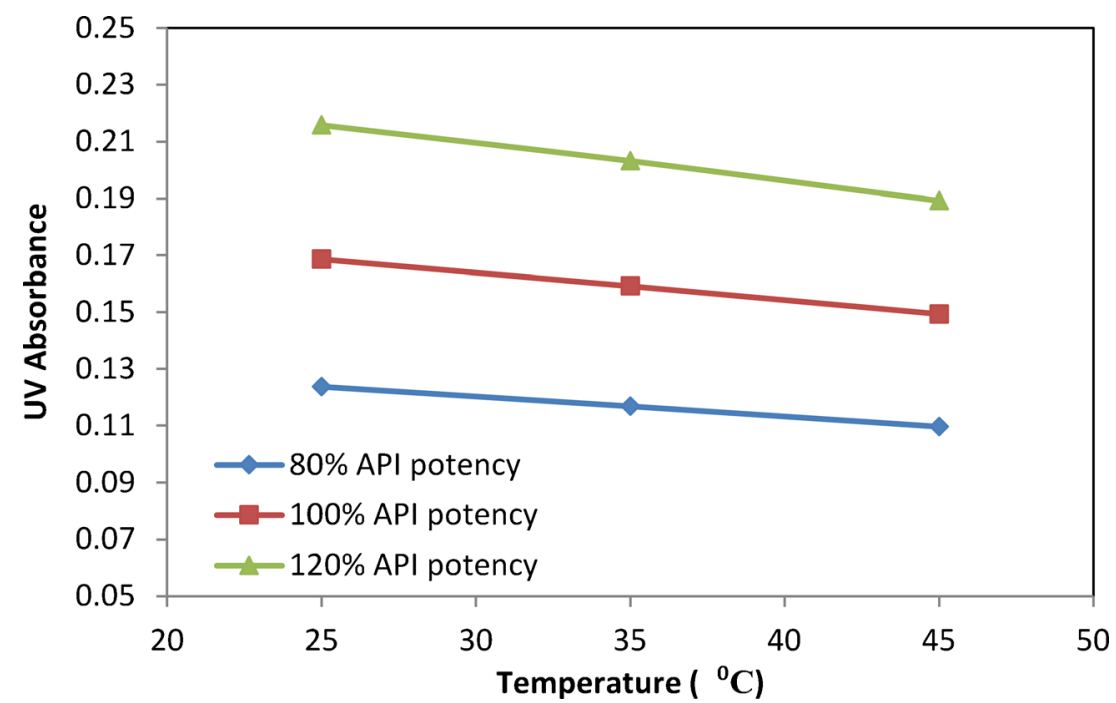

Fig. 8. ATR-UV absorbance values $(340 \mathrm{~nm})$ on three samples of different concentration and at three different temperatures 
Table I. Measured Refractive Index Values on API Solution ( $100 \%$ Label Claim) Under Different Temperature

\begin{tabular}{lc}
\hline Temperature $\left({ }^{\circ} \mathrm{C}\right)$ & Measured refractive index \\
\hline 21.9 & 1.4739 \\
24.0 & 1.4722 \\
25.9 & 1.4694 \\
27.9 & 1.4674 \\
29.8 & 1.4660 \\
\hline
\end{tabular}

temperature. Since liquids and solutions become less dense at higher temperatures light passing through higher-temperature samples encounter fewer solute and solvent molecules. When the $100 \%$ API fill solution was measured for solution density, it was found that it decreases about $2 \%$ with temperature increasing from 25 to $45^{\circ} \mathrm{C}$. Theoretically, this would result in the same degree of decrease in UV absorbance if density change is the only contributing factor to the absorbance change. Clearly, there must be some other factor that accounts for most of the ATR-UV absorbance with increased temperature.

In addition to the effect on the density of the API solution, temperature can also cause the change of the refractive index of the sample solution and ATR probe. In ATR measurement, the depth of penetration of light at a fixed wavelength is directly related to the refractive index of both sample and ATR material according to Eq. 1 . The $d_{p}$ property is actually the depth that the evanescent wave's electric field decays to $1 / e$ of the signal at the surface.

$d_{p}=\frac{\lambda}{2 \pi\left(n_{1}^{2} \sin ^{2} \theta-n_{2}^{2}\right)^{1 / 2}}$

Where $\lambda$ is the wavelength of light, $\theta$ is the ATR angle of incidence of the light relative to the crystal surface, $\mathrm{n}_{1}$ is the refractive index of the ATR crystal and $n_{2}$ is the refractive index of the sample medium (23). Reducing the refractive index of the sample results in a shorter effective pathlength and the absorbance will be commensurately lower. The sensitivity of the refractive index change of sapphire with temperature is minimal and often negligible. However, the change of the refractive index of liquids can be significant as the temperature changes. The refractive index of the fill solution of $100 \%$ potency was measured in the range from 20 to $30^{\circ} \mathrm{C}$. As shown in Table I, the refractive index values decreased with increasing temperature. Based on these results, a $10^{\circ} \mathrm{C}$ shift in temperature during an experiment would result in a calculated drop in pathlength of $\sim 5 \%$, for a sapphire ATR crystal (with a refractive index of 1.8 at $340 \mathrm{~nm}$ ) and an ATR incidence angle of $60^{\circ}$. It can be concluded that the observed drift of the ATRUV dissolution profile was caused by the temperature change, mostly related to the pathlength change resulting from temperature-induced change of refractive index of the API solution.

As an attempt to improve robustness of the ATR-UV model against temperature, multivariate calibration methodology was also explored using the same calibration data. Different data preprocessing techniques were investigated to manage the pathlength changes including standard normal variate (SNV) and multiplicative signal correction (MSC). However, no significant improvement was found. In addition, since the excipients solution was used as the background, all the features in the UV absorbance spectra are related to the API. As the UV data so far were collected by using the solvent solution as a background, the obtained UV spectra only represent the API features. By introducing excipient absorbance features into the spectra it is hypothesized that the excipients peaks could be used as an internal reference as was done on the Raman data, ATR-UV spectra were also collected for the calibration samples using air as the background. Figure 9 shows the comparison of the UV spectra of the API solution at $100 \%$ of label claim obtained by using the two different backgrounds. The UV spectrum of excipient solution by using air as background is also included. It can be seen

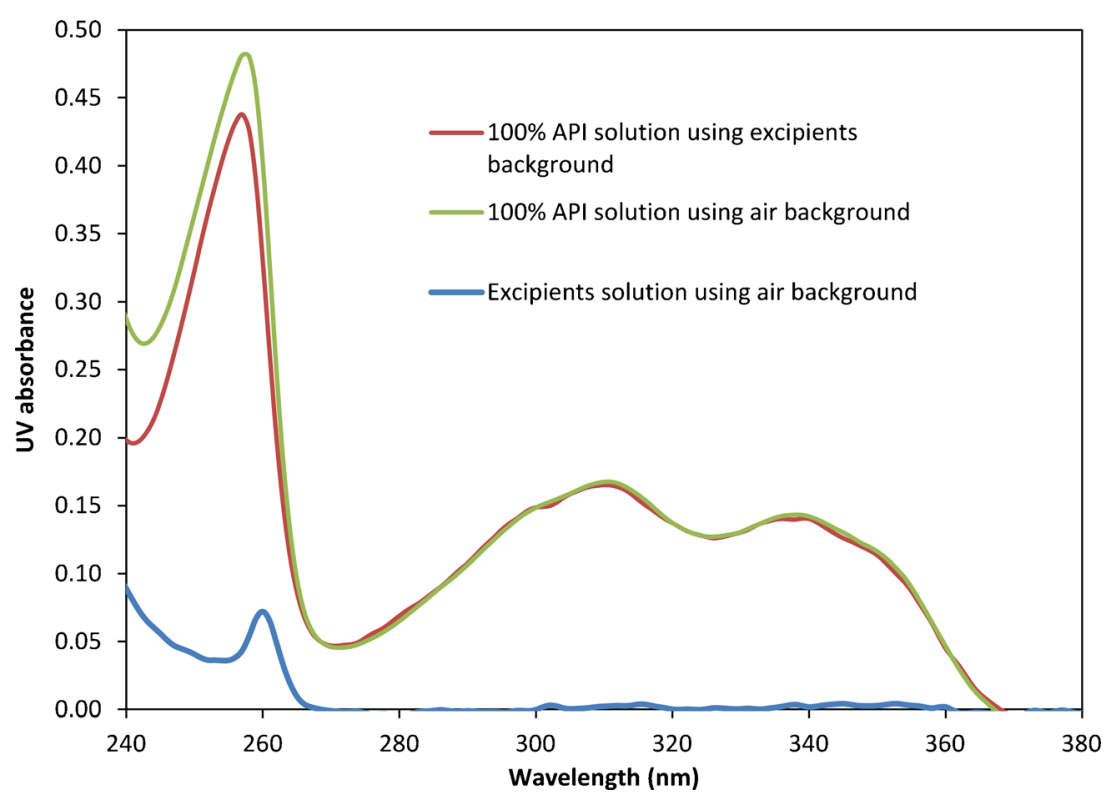

Fig. 9. UV absorbance spectra of sample solution $\left(\sim 100 \%\right.$ label claim, $\left.\sim 25^{\circ} \mathrm{C}\right)$ by using air and excipients background and spectrum of excipient solution by using air background 


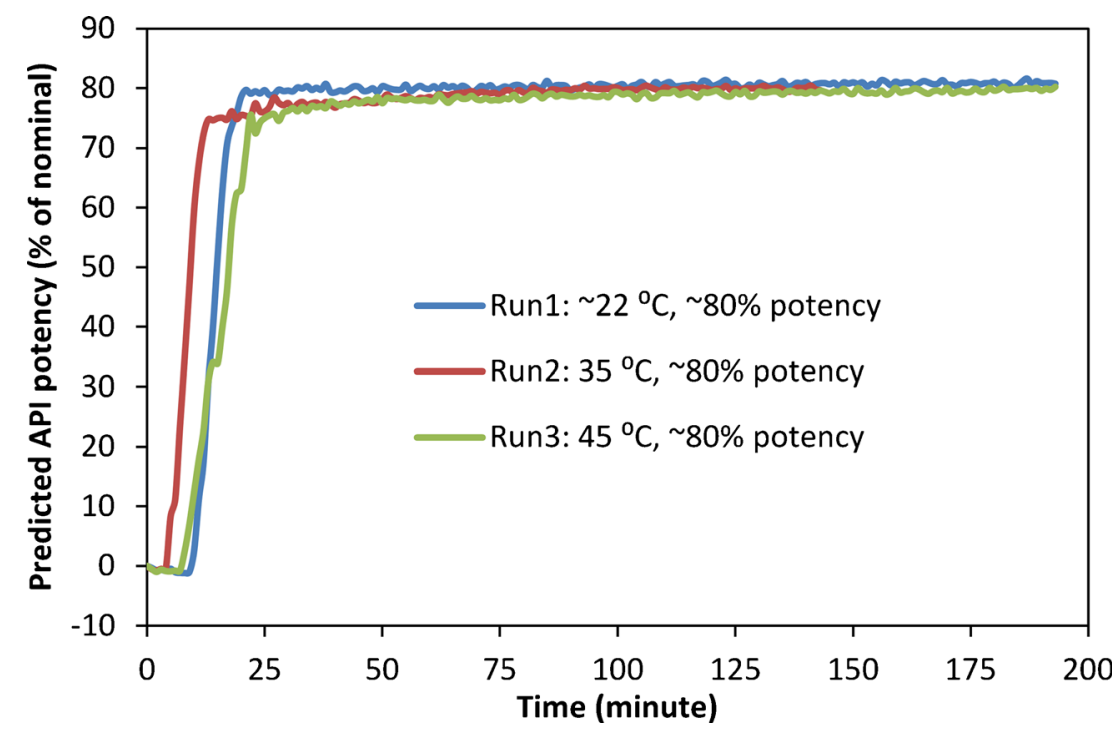

Fig. 10. Predicted API dissolution profiles by Raman method on three batches ( $\sim 80 \%$ label claim) run under three different temperatures

that, the two spectra of $100 \%$ API solution are very similar except absorbance intensity difference for the band around $250 \mathrm{~nm}$. It is clear that the excipient UV signal is very weak and overwhelmed by the strong absorbance of API so that no unique features can be used for normalization.

As a comparison, robustness of the Raman method against temperature was also investigated. Figure 10 shows the profile of Raman prediction on an $80 \%$ API solution different temperatures, demonstrating no observable sensitivity over the $30^{\circ} \mathrm{C}$ span of the experiment. This is expected since the Raman probe is, firstly, not an ATR probe; and secondly, the internal standardization with the solvent peaks would remove any bulk changes that impact the overall solution. This observation was supported by additional in-line studies.

\section{CONCLUSIONS}

Both ATR-UV and Raman methods have been investigated for in-line quantitative determination of the API potency and monitoring the API dissolution profile during the manufacturing process of a SGC product. With easy instrumental setup and simple data processing, both techniques were able to provide trending information about API dissolution in the process.

For quantification purposes, the ATR-UV method was found to be sensitive to temperature change of the solution as a consequence of the change in the refractive index of the solution and the consequent change in pathlength that could not be overcome by direct data processing. On the other hand, the Raman method was found to be able to give accurate and robust prediction of API potency in various process conditions through the use of a spectral normalization step. The method was proved to be robust against temperature change, stirring and other process variations.

\section{ACKNOWLEDGMENTS}

The authors would like thank Pankaj Shah from BristolMyers Squibb for their support and assistance during preparation of the manuscript.

\section{REFERENCES}

1. Benza HI, Munyendo WLL. A review of progress and challenges in soft gelatin capsules formulations for oral administrtion. Int J Pharm Sci Rev Res. 2011;10(1):20-4.

2. Reddy G, Muthukumaran M, Krishnamoorthy B. Soft gelatin capsules-present and future prospective as a pharmaceutical dosage forms-a review. Int J Adv Pharm Gen Res. 2013;1(1):20-9.

3. Reddy BV, Deepthi A, Ujwala P. Capsule production-industrial view. J Glob Trends Pharm Sci. 2012;3(4):887-909.

4. Allen LV, Popovich NG, Ansel HC. Ansel's pharmaceutical dosage forms and drug delivery systems. 9th ed. Philadelphia: Lippincot Williams \& Wilkins; 2011.

5. Santos H. Manufacturing and quality control of liquid-filled twopiece hard capsules. Int Pharm Ind. 2012;4(1):82-4.

6. FDA, Guidance for industry, Q8 (R2) pharmaceutical development. 2007. http://www.fda.gov/downloads/Drugs/ GuidanceComplianceRegulatoryInformation/Guidances/ ucm073507.pdf.

7. FDA, Guidance for Industry PAT - a framework for innovative pharmaceutical development, manufacturing, and quality assurance. 2004. http://www.fda.gov/downloads/Drugs/ GuidanceComplianceRegulatoryInformation/Guidances/ ucm070305.pdf.

8. Beer TD, Burggraeve A, Fonteyne M, Saerens L, Remon JP, Vervaet C. Near infrared and Raman spectroscopy for the inprocess monitoring of pharmaceutical production processes. Int $\mathrm{J}$ Pharm. 2011;417:32-47.

9. Flaten GR, Ferreira AP, Bellamy L, Frake P. PAT within the QbD framework: real-time end point detection for powder blends in a compliant environment. J Pharm Innov. 2012;7:38-45.

10. Chen CS, Alvarado JM. On-line monitoring of industrial-scale blending processes using NIR: an overview from system selection to measurement in GMP environments. Am Pharm Rev. 2007;10(4):22-9.

11. Singh R, Román-Ospino $A D$, Romañach $R J$, Ierapetritou $M$, Ramachandran R. Real time monitoring of powder blend bulk density for coupled feed-forward/feed-back control of a continuous direct compaction tablet manufacturing process. Int J Pharm. 2015;495(1):612-25.

12. Fevotte G. In situ Raman spectroscopy for in-line control of pharmaceutical crystallization and solids elaboration processes: a review. Chem Eng Res Des. 2007;85(7 A):906-20.

13. Liang JK, Byrn S, Newman A, Stults J, Smolenskaya V. On-line Raman spectroscopy in pharmaceutical process development: application and future prospects. Am Pharm Rev. 2007;10(3):45-51. 
14. Saerens L, Segher N, Vervaet C, Remon JP, Beer TD. Validation of an in-line Raman spectroscopic method for continuous active pharmaceutical ingredient quantification during pharmaceutical hot-melt extrusion. Anal Chim Acta. 2014;806:180-7.

15. Jansari SK, Patel NB, Patel P, Desai HT. development and validation of cleaning procedure of mixing equipment used for manufacturing ciprofloxacin HCL and Tinidazole tablet by using UV spectroscopy. J Pharm Sci Innov. 2012;1(4):9-12.

16. Matias R, Ribeiro PRS, Sarraguca MC, Lopes JA. A UV spectrophotometric method for the determination of folic acid in pharmaceutical tablets and dissolution tests. Anal Methods. 2014;6:3065-71.

17. Lu X, Lozano R, Shah P. In situ dissolution testing using different UV fiber optic probes and instruments. Dissolut Technol. 2003;11:6-15.

18. Rathore N, Qi W, Ji W. Cleaning characterization of protein drug products using UV-vis spectroscopy. Biotechnol Prog. 2008;24(3):684-90.

19. Inman GW, Wethington E, Baughman K, Horton M. System optimization for in situ fiber-optic dissolution testing. Pharm Technol. 2001;10:92-100.

20. Guillot A, Limberger M, Krämer J, Lehr CM. In situ drug release monitoring with a fiber-optic system: overcoming matrix interferences using derivative spectrophotometry. Dissolut Technol. 2013;20(2):15-9.

21. Liauw MA, Baylor LC, O'Rourke PE. UV-visible spectroscopy for on-line analysis. In: Bakeev KA, editor. Process analytical technology: spectroscopic tools and implementation strategies for the chemical and pharmaceutical industries. John Wiley \& Sons; 2010. p. 81-106.

22. Mirza T, Liu Q, Vivilecchia R, Joshi Y. Comprehensive validation scheme for in situ fiber optics dissolution method for pharmaceutical drug product testing. J Pharm Sci. 2009;98(3):1086-94.

23. Billot P, Couty M, Hosek P. Application of ATR-UV spectroscopy for monitoring the crystallisation of UV absorbing and nonabsorbing molecules. Org Process Res Dev. 2010;14:511-23.
24. Simone E, Saleemi AN, Tonnon N, Nagy ZK. Active polymorphic feedback control of crystallization processes using a combined Raman and ATR-UV/Vis spectroscopy approach. Cryst Growth Des. 2014;14:1839-50.

25. Liotta V, Sabesan V. Monitoring and feedback control of supersaturation using ATR-FTIR to produce an active pharmaceutical ingredient of a desired crystal size. Org Process Res Dev. 2004;8(3):488-94.

26. Reimann S, Knopke LR, Spannenberg A, Langer P, Bentrup U. Identification of reaction intermediates in $\mathrm{AlCl} 3$-mediated cyclocondensation reactions by simultaneous in situ ATR-FTIR and UV-vis spectroscopy. Tetrahedron. 2013;69(15):3338-47.

27. Salepour S, Dube MA. Reaction monitoring of glycerol stepgrowth polymerization using ATR-FTIR spectroscopy. Macromol React Eng. 2012;6(2-3):85-92.

28. Subedi DP, Adhikari DR, Joshi UM, Poudel HN, Niraula B. Study of temperature and concentration dependence of refractive index of liquids using a novel technique. Kathmandu Univ J Sci Eng Technol. 2006;II(1):1-7.

29. Chang C-F, Wang S-C, Shigeto S. In situ ultra low-frequency raman tracking of the polymorphic transformation of crystalline 1,1'-binaphthyl. J Phys Chem C. 2014;118:2702-9.

30. Walker GM, Greene K, Zhai H, Jones DS, Andrews GP. The application of in-situ Raman spectroscopy to pharmaceutical fluidized bed granulation processes Raman. Am Pharm Rev. 2009;12(2).

31. Wray PS, Sinclair WE, Jones JW, Clarke GS, Both DA. The use of in situ near infrared imaging and Raman mapping to study the disproportionation of a drug $\mathrm{HCl}$ salt during dissolution. Int $\mathrm{J}$ Pharm. 2015;493:198-207.

32. Kogermann K, Penkina A, Predbannikova K, Jeeger K, Veski P, Rantanen J, et al. Dissolution testing of amorphous dispersions. Int J Pharm. 2013;444:40-6.

33. Tres F, Treacher K, Booth J, Hughes LP, Wren SAC, Aylott JW, et al. Real time Raman imaging to understand dissolution performance of amorphous solid dispersions. J Control Release. 2014;188:53-60. 